\title{
Particle Catcher Using Induced-Charge Electroosmosis
}

\author{
Hideyuki Sugioka* \\ Department of Mechanical Systems Engineering, \\ Shinshu University 4-17-1 Wakasato, Nagano 380-8553, Japan
}

(Dated: November 10, 2016)

\begin{abstract}
Finding an innovative separation mechanism is a central task in future microfluidic systems. We propose a size-controllable microfluidic catching device that has a face-to-face structure consisting of elastic beams that change the acceptable particle size dynamically by hydrodynamic force due to induced charge electroosmosis (ICEO) in water and numerically examine the novel separation mechanism consisting of catching and releasing motions with size selectivity. By an implicit strongly coupled simulation technique between a fluid and an elastic structure based on the boundary element method, along with the thin double-layer approximation, we find that the catching device works effectively at low applied voltages in a realistic microfluidic channel and shows a wide range dynamic size selectivity. Furthermore, by modeling the ICEO phenomena with elastic motion, we successfully explain the acceptable particle size of the catching device. We believe that our proposed device will contribute to realizing innovative microfluidic systems in the future.
\end{abstract}

\footnotetext{
* sugioka.hideyuki@canon.co.jp
} 


\section{INTRODUCTION}

Innovative separation mechanisms of chemical substances (e.g., biomolecules and hazardous materials) are always required for promising microfluidic systems such as a lab-ona-chip and micro-total-analysis systems ( $\mu$ TASs) [1] since natural samples such as food, drinking water, blood, oral/fluid, and urine are usually complex. Thus, various separation techniques have been proposed and examined in the microfluidic field [2], e.g., from fundamental physics, Brownian ratchet [3], obstacle array [e.g., deterministic lateral displacement] [4], side channel (e.g., hydrodynamic filtration) [5], external force (e.g., acoustic and magnetic separation) [6], and trapping force (e.g., optical tweezers) [7] have been proposed for separation. In particular, the obstacle array has attracted much attention since it changes the flow direction of target particles in the main stream in the channel passively and simply; for example, Duke and Austin [8] theoretically showed that a sieve consisting of a periodic array of oblong obstacles can separate biological micromolecules; Davis et al. [9] experimentally showed that circular obstacles can fractionate whole blood components. In addition, Morton et al. [10] experimentally showed that an obstacle array can be used for a novel biological processing method that moves selected particles across functional laminar streamlines in pressure-driven microfluidic flow; they demonstrated on-chip cell treatments such as labeling and washing, and bacterial lysis and chromosomal extraction. Since the method enables sequential chemical operations, it is definitely important.

However, time-consuming manual separation is still used in practice; for example, separation using a centrifugal machine with a disposable sample preparation kit including several reagents and glass fibers for adsorption is used as the standard sample preparation before the polymerase chain reaction process for viral deoxyribonucleic acid (DNA) or ribonucleic acid (RNA) detection. Thus, from the industrial side, microfluidic systems based on the conventional method are explored extensively. Chung et al. [11] reported that a marine norovirus in a single oyster was detected within $4 \mathrm{~h}$ by using $\mu$ TAS to perform automatically a series of essential processes, such as cell concentration, lysis (RNA extraction), nucleic acid amplification, and detection. Note that in their approach, charge-switchable microglass beads are used instead of glass fibers in the ordinary manual process and play a central role; i.e., viral materials are separated through the surface adsorption of the beads and a chemical process is operated sequentially by conveying the beads through the flow channel. Although 
the system shows marked improvement, $4 \mathrm{~h}$ is insufficient to cause real innovation; thus, in addition to the miniaturization of large peripheral equipment, more rapid and efficient continuous-flow processing is strongly required.

For this requirement, ciliumlike bio-inspired microfluidic devices having curled elastic filaments or hairlike elastic rods are explored extensively [12], since microorganisms use cilia to catch suspended particles from surrounding fluids and the catching motion is vital to maintain their living systems $[13,14]$; i.e., similar to cilia, controllable catching and releasing motions are considered essential to realize a faster process to deal with various chemical substances even in microfluidic systems. Bhattacharya et al. [13] theoretically showed that, by assuming an adhesive interaction of cilial tips with a particle's surface, the particle can be released, propelled, or trapped by the cilia; Tripathi et al. [14] showed that the particle size selectivity arises as a result of adhesive and hydrodynamic interactions. However, since those devices are usually driven by large magnetic or electric equipment [12], they are not suitable for miniaturization; in addition, the aim of nature is usually different from that of our individual microfluidic system. For example, using a passive adhesive surface for catching is not a good strategy at least for high-throughput processing in microfluidic systems since it requires a stronger swing to release the particle [14]. Thus, for miniaturization, developing a size-selectable particle catcher based on a different fundamental key technology that enables a low-voltage drive, high-density fabrication, and high-throughput processing is required.

Recently, induced-charge electroosmosis (ICEO) [15-19] caused by the interaction between an electric field and ions in an electric double layer formed by the polarizing effect has been considered to be a key concept for innovative microfluidic devices [15, 20], e.g., a pump $[15,21]$, a valve $[22,23]$, and a mixer $[15,24]$, since it causes a large flow $(\sim 1$ $\mathrm{mm} / \mathrm{s})$ because of the large zeta potential $(\sim 1 \mathrm{~V})$ under ac electric fields $(<\sim 1 \mathrm{kHz})$ and it preferably suppresses the dc electric field trouble due to the chemical reaction and the generation of bubbles. In particular, Gangwal et al. [25] observed the motion of a halfcoated metal sphere using ICEO. Ziebert et al. [19] theoretically showed that the behavior of a conductive membrane in a static electric field is also related to ICEO. Furthermore, we proposed an elastic valve using ICEO and numerically showed that it functions effectively at high frequency with low applied voltages [23]. Note that ICEO includes the concept of ac electro-osmosis (ACEO) discovered by Ramos et al. [16, 17, 26], who first showed that, theoretically, the accumulated electric charge in the electric double layer of the pair elec- 
trodes can generate a large flow field by the coulomb force with the existence of a tangential electric field [26] and experimentally observed the velocities of the vortex flow [16]. Bazant and Squires showed how general the ACEO phenomenon is and termed it ICEO [15]. In addition, we also propose an elastic beating pump using ICEO and numerically showed that it produces a remarkably large peak flow velocity $(\sim 7 \mathrm{~mm} / \mathrm{s}$ at $2 \mathrm{~V})[27]$.

Obviously, ICEO is suitable for realizing a particle catcher in microfluidic systems since it enables a low voltage drive, high-density fabrication, and high-throughput processing. Furthermore, catching particles in a viscous fluid is a difficult task; for example, the approaching of an elastic beam to a target particle usually induces the motion of the particle going away because of hydrodynamic interactions. Furthermore, obtaining a controllable size selectivity for rapid processing with a novel sequential operation is an indispensable task for future promising microfluidic applications, as mentioned before. Thus, it is challenging to consider a size-selectable particle catcher using ICEO in a viscous fluid with a novel sequential rapid operation. To overcome those problems, we consider a face-to-face structure consisting of elastic beams that change the acceptable particle size dynamically by hydrodynamic force due to ICEO in water, in addition to using a vortex flow due to the upward motion of the elastic beam edges to withdraw a suspended particle into the catching device region. Note that the face-to-face structure plays a role of a dipping chamber for chemical processing, and the catching and releasing behavior of a particle can provide an ideal sequential operation for labeling, washing, and lysis; i.e., in this study, we focus on an elastic particle catcher using ICEO with a face-to-face elastic beam structure and elucidate its design concept with the concept of novel sequential processing. In particular, by using the implicit strongly coupled simulation technique [23] based on the boundary element method (BEM), we analyze the microfluidic particle catcher using ICEO and clarify the catching behavior.

\section{THEORY}

\section{A. Particle catcher using a face-to-face elastic beam configuration}

Figure 1 shows a schematic view of an elastic particle catcher using induced-charge electroosmosis (ICEO). As shown in Fig. 1(c), we propose a particle catcher using a face-to-face elastic beam configuration with the ICEO phenomena; i.e., we consider a rectangular channel 


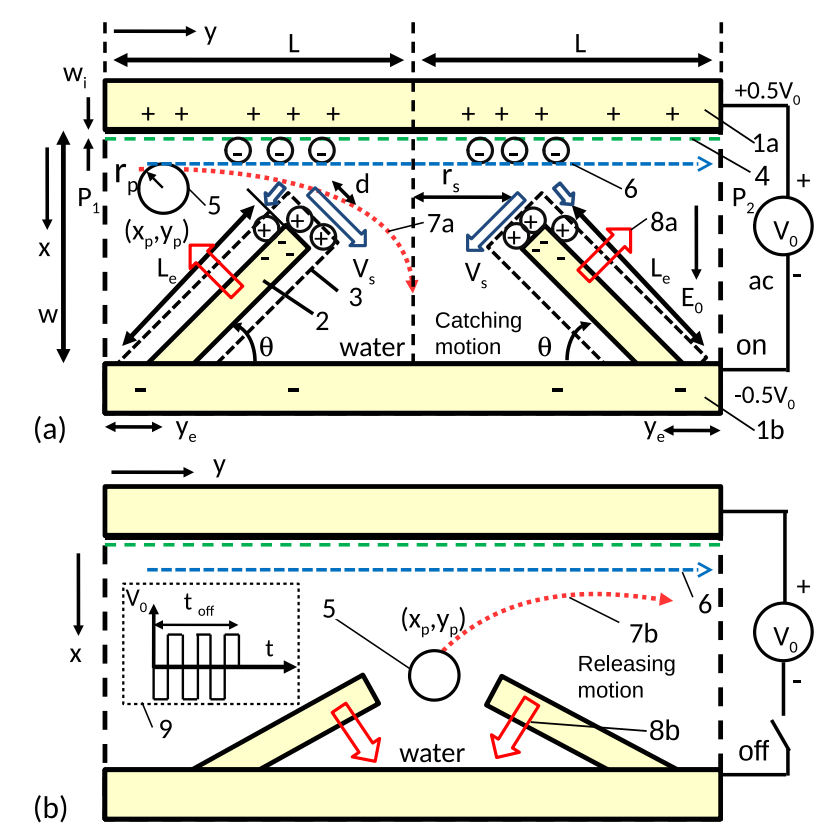

FIG. 1. (Color online) Schematic view of an elastic particle catcher using induced-charge electroosmosis (ICEO). 1a: upper electrode, 1b: lower electrodes, 2: conductive elastic beam, 3: electric double layer, 4: insulation layer, 5: particle, 6: main stream, 7a: catching trajectory, 7b releasing trajectory, 8a: up-swing motion of elastic beam, 8b: down-swing motion of elastic beam, and 9: driving voltage. We place a pair of conductive elastic beams of length $L_{e}=1.5 \mathrm{w}$ and width $d=0.07 w$ on the lower electrode in a rectangular channel of length $2 L=4.5 w$ and width $w=100$ $\mu \mathrm{m}$. Here, typically, the applied voltage $V_{0}$ is 1.2 to $1.7 \mathrm{~V}$, the pressure difference $\Delta P\left(\equiv P_{2}-P_{1}\right)$ is $0 \mathrm{~Pa}$, and $y_{m}=0.7 w$ is the beam bottom positions. The Poisson ratio $\nu$ and the shear modulus $G$ of the elastic beams are 0.5 and $500 \mathrm{kPa}$, respectively, and the viscosity $\mu$ is $1 \mathrm{mPa}$.

(of length $2 L=4.5 w$ and width $w=100 \mu \mathrm{m}$ ) having parallel electrodes and typically place a pair of conductive elastic beams of length $L_{e}=1.5 \mathrm{w}$ and width $d=0.07 \mathrm{w}$ on the lower electrode at a $20^{\circ}$ tilt angle $\theta$ at $y=y_{e}$ and $y=2 L-y_{e}$ [in Fig. 1(d)]. To draw a particle at $(x / w, y / w)=(0.3, L)$ (above the device) into the device region, we apply an ac electric voltage $V_{0}$ between the electrodes [in Fig. 1(c)] and turn off the electric voltage at $t=t_{\text {off }}$ [in Fig. 1(c)] to release the particle from the device region to the outer stream region. Here, we assume that the period of the ac frequency is much smaller than $t_{\text {off }}$; thus, we can expect that the occurrence of chemical reactions is prevented; in fact, the relatively high applied voltage condition (e.g., $V_{0}=3 \mathrm{~V}$ ) is often common in ACEO experiments [20]. 


\section{B. Numerical method}

Simulations of our problem (i.e., particle catcher) are difficult because our problem is a fluid solid interaction (FSI) problem, which has strong nonlinearity and multidisciplinary nature. For example, in the finite element method (FEM), the elastic problem is usually described by the global stiffness equation $[K]\{u\}=\{F\}$, where $[K]$ is the global stiffness matrix, $\{u\}$ is the global node displacement vector, and $\{F\}$ is the global force vector. For the current system, even though Lame's constants are assumed to be independent of the structure, the nonlinearity related to $[K]$ and $\{F\}$ remains because $[K]$ and $\{F\}$ strongly depend on the structure in our large deflection problem of the solid beam. Furthermore, our fluid problem also has a strong nonlinearity because of the change in both the channel structure and the exerted force owing to the motion of beams, even though we use the Stokes equation. That is, our problem requires sophisticated treatment for both elastic and fluid problems. In particular, the most intrinsic difficulty of our problem is that the boundary conditions both for a fluid and a solid change over time, and in addition, the two fields are expressed by related but different quantities, velocity and displacement. This results in the difficulty in the precise treatment of the boundary between two fields. This is the true difficulty of our current problem.

Furthermore, from the viewpoint of the numerical calculation method, our problem is a free boundary problem, which changes the boundary position in time, and the change in the boundary position often causes inaccurate calculations. For example, FEM often causes inaccuracy owing to the deformation of meshes or the switching of mesh typologies, while the finite difference method and other Eulerian methods also cause inaccuracy owing to the difficulty in determining the precise boundary position in the fixed Eulerian meshes. Moreover, a weak coupled FSI calculation method that calculates a fluid and solid alternatively often causes serious instability. In addition, we need to consider ICEO flow velocities around a beam in our problem. Thus, our problem is highly challenging as a numerical simulation problem. However, we overcome all of the difficulties in solving the problem in the current system by using the strongly coupled simulation technique that was previously developed [23]. This is the advantage of this work, i.e., by using the sophisticated method described in Ref. [23], we first succeeded in the reliable simulations of the motion of the particle catcher. Namely, on the basis of the the boundary element method (BEM) along with an implicit 
strong coupled method between a fluid and a solid, we here perform a highly accurate meshless FSI calculation of the current system and clarify the background physics of the particle catcher numerically.

Specifically, on the basis of the FSI method that we developed [23], we calculate the flow fields and deflections of the beams using the Steady Stokes equations of fluid and the Steady Navier's equations of solid:

$$
\begin{aligned}
& \mu \nabla^{2} \dot{\boldsymbol{u}}-\nabla p=0, \quad \nabla \cdot \dot{\boldsymbol{u}}=0 \\
& \left(\lambda_{e}+\mu_{e}\right) \nabla(\nabla \cdot \boldsymbol{u})+\mu_{e} \nabla^{2} \boldsymbol{u}=0
\end{aligned}
$$

where $p$ is pressure, $\mu(\sim 1 \mathrm{mPa}$ s) is viscosity, $\dot{\boldsymbol{u}}(=\boldsymbol{v})$ is velocity, $\boldsymbol{u}$ is displacement, and $\mu_{e}$ and $\lambda_{e}$ are Lame's constants on solid. Furthermore, we use the boundary condition at the interface between the fluid and the elastic beams as follows:

$$
\begin{gathered}
\dot{\boldsymbol{u}}=\tilde{\boldsymbol{v}}+\boldsymbol{V}_{\mathrm{s}} \\
\boldsymbol{f}_{e}\left(=\boldsymbol{f}_{e}^{\prime}+\boldsymbol{f}^{d e p}+\boldsymbol{f}^{r e p}\right)=\boldsymbol{f}_{f}
\end{gathered}
$$

where $\tilde{\boldsymbol{v}}$ is the velocity on the inside edge of the electric double layer (EDL), $\boldsymbol{f}_{e}$ and $\boldsymbol{f}_{f}$ are the total surface traction vectors (defined by the opposite normal vectors) of the beam and fluid, respectively, $\boldsymbol{f}_{e}^{\prime}, \boldsymbol{f}_{e}^{d e p}$, and $\boldsymbol{f}_{r e p}$ are the surface tractions due to the deflection of solid, the dielectrophoresis (DEP), and the phenomenologically introduced repulsion force [see Eq. B5 in Appendix B], respectively. Note that, before recognizing the importance of the ICEO flows around the metal, DEP is the main effect that generates the external force and torque on the metal in water. Thus, we consider the DEP effects in our calculations, and we can calculate the DEP traction resulting from the Maxwell stress as $\boldsymbol{f}^{d e p}=-\frac{1}{2} \epsilon \boldsymbol{E}_{s}^{2} \boldsymbol{n}$. However, nowadays, it is recognized that the contribution of the DEP effects is just $20 \%$ in the bulk region [28] and it is often negligible near the electrode compared with that of the ICEO effect [22]. Furthermore, by solving the Laplace equation under the Neumann and Dirichlet boundary condition, we calculate the slip velocity $\boldsymbol{V}_{s}$ at each time step on the basis of the Helmholtz-Smoluchowski formula [Eq. A1 in Appendix A]. Note that the neglect of an unsteady term in the Stokes equation and charging time were discussed in detail and our calculation method was justified in Ref. [23]. 


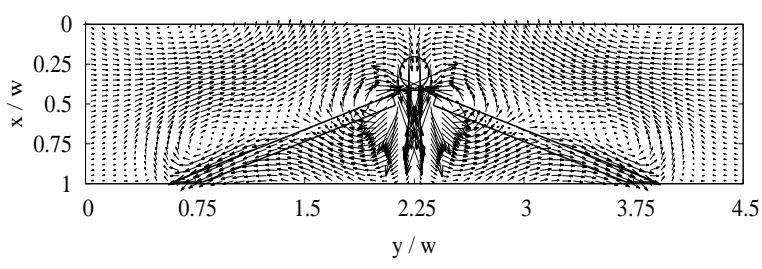

(a) Flow field at $t / T_{0}=0\left(r_{p} / w=0.1\right)$

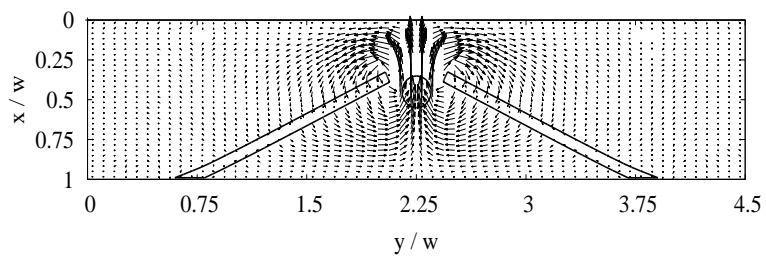

(c) Flow field at $t / T_{0}=12\left(r_{p} / w=0.1\right)$

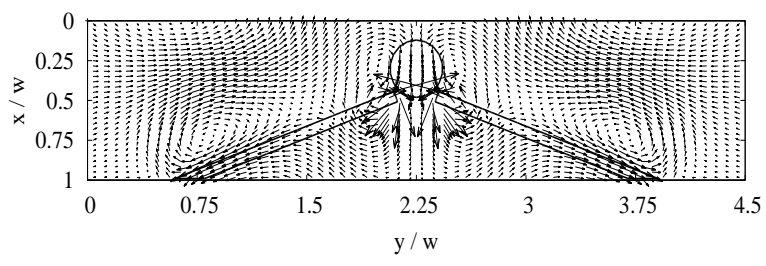

(e) Flow field at $t / T_{0}=0\left(r_{p} / w=0.18\right)$

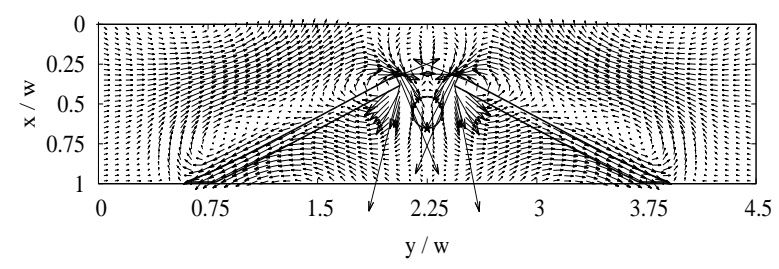

(b) Flow field at $t / T_{0}=8\left(r_{p} / w=0.1\right)$

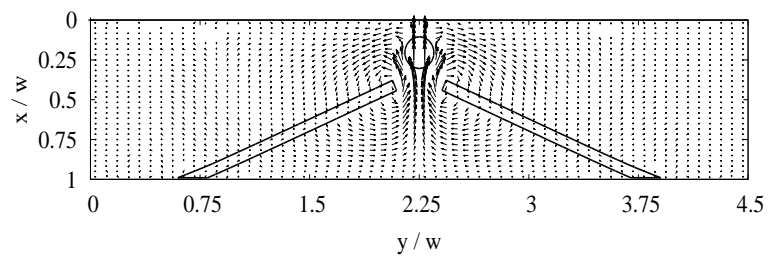

(d) Flow field at $t / T_{0}=20\left(r_{p} / w=0.1, \times 1.5\right)$

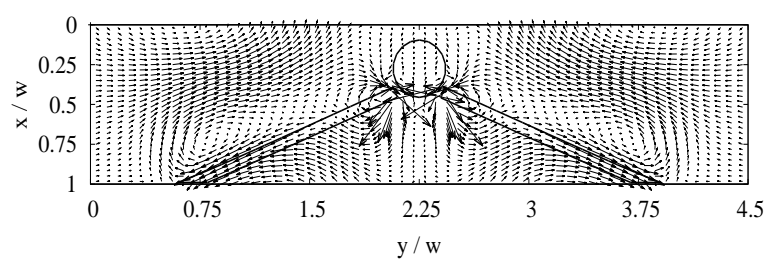

(f) Flow field at $t / T_{0}=8\left(r_{p} / w=0.18\right)$

FIG. 2. Catching and releasing motions using ICEO with elastic beams at $G=0.5 \mathrm{MPa}$ at $V_{0}=1.5 \mathrm{~V}$. Here, the initial position of the particle is $\left(x_{p} / w, y_{p} / w\right)=(0.3,2.25), t_{o f f} / T_{0}=10$, $d_{\text {min }} / w=0.05, r_{s}=L-y_{e}-L_{e} \cos \theta=0.14, L / w=2.25, y_{e} / w=0.7, \nu=0.5, L_{e} / w=1.5$, $d / w=0.07, \theta=20^{\circ}, w=100 \mu \mathrm{m}, T_{0}=1 \mathrm{~ms}, \mu=1 \mathrm{mPa}$ s, and $\Delta P=0 \mathrm{~Pa}$. Note that in $(\mathrm{d})$, we magnify the scale of flow vectors by 1.5 since they are too small compared with those of the other figures.

\section{RESULTS}

\section{A. Catching and releasing motions}

Figure 2 shows the catching and releasing motions of our device when $V_{0}=1.5 \mathrm{~V}$, $t_{o f f} / T_{0}=10, d_{\min } / w=0.05, r_{s}=0.14, L / w=2.25, y_{e} / w=0.7, \nu=0.5, G=0.5 \mathrm{MPa}$, $L_{e} / w=1.5, d / w=0.07, \theta=20^{\circ}, w=100 \mu \mathrm{m}, T_{0}=1 \mathrm{~ms}, \mu=1 \mathrm{mPa} \mathrm{s}$, and $\Delta P=0$ Pa. In Fig. 2(a), initially (at $t / T_{0}=0$ ), we observe two vortex flows due to ICEO below the target particle of radius $r_{p}=0.1 w$, and the vortex flows pull the particle into the device region. As a result, we find the particle in the device region at $t / T_{0}=8[$ Fig. 2(b)]. Here, 
we also observe the upward motion of the edge of the elastic beams; because of this motion, the particle is pulled into the device region. Then, by turning off the applied voltage at $t=t_{\text {off }}\left(=10 T_{0}\right)$, we observe the significant upward flow at $t / T_{0}=12[$ Fig. $2(\mathrm{c})]$; this upward flow pops up the particle. Thus, the particle is released into a main stream region in a channel at $t / T_{0}=20$ [Fig. $2(\mathrm{~d})$ ]. Here, the upward flow is generated by the downward motion of the beam edges owing to the elastic force. Furthermore, in Figs. 2(e) and 2(f), we find that a large particle $\left(r_{p} / w=0.18\right)$ cannot enter the device region because of the geometrical condition, although the acceptable particle radius can be changed to some extent by changing the applied voltages, as explained in Sec. II-C.

\section{B. Size and time controllability}

Figure 3 shows the controllability of the keeping time and acceptable size. Here, $x_{p}$ is the $x$ position of the particle, $h_{m} \equiv x_{p}^{\max }-x_{p}^{t=0}$ is the maximum catching distance, and $t_{w}$ is the keeping (working) time defined as a period that satisfies the condition $h(t) \equiv x_{p}^{t=t}-x_{p}^{t=0} \geq$ $0.1 w$. Note that $t_{w}$ is introduced as a simple mean theory to evaluate complex phenomena qualitatively. In detail, we consider that the typical radius is $r_{p} \simeq 0.1 w$, the typical initial particle position is $x_{p} \simeq 0.3 w$, and the typical initial beam edge position is $x_{b} \simeq 0.5 w$. Thus, if $h \geq 0.1 w$, the bottom of the particle is lower than the beam edge on average. This is why we consider the condition $h(t) \equiv x_{p}^{t=t}-x_{p}^{t=0} \geq 0.1 w$ to define $t_{w}$. Although the definition of $t_{w}$ is too simple, we believe that it is useful for evaluating a complex system that includes many parameters.

Figures 3(a), 3(c), and 3(e) show that $t_{w}$ is controlled in the range $5 \leq t_{w} / T_{0} \leq 15$ by adjusting the switch-off time $t_{\text {off }}$ with the considerable catching distance in the range $0 \leq h_{m} / w \leq 0.3$. Specifically, in Fig. 3(a), the particle starts to move toward the upper direction at $t / T_{0} \simeq 8$ after the particle enters in the trapped region, and it can happen before $t=t_{\text {off }}$. Namely, when $t_{\text {off }}$ is sufficiently large, the particle is trapped but is automatically released; thus, in the steady state, the particle cannot remain in the trapped region. This is because the downward ICEO flow at the beam edge position can no longer be accumulated in the chamber region (trapped region) because of the stoppage of the beams in the steady state, as explained in detail in Appendix B1. Furthermore, for the same reason, $t_{w}$ saturates at a large $t_{\text {off }}$ when $V_{0}$ is large, as shown in Fig. 3(e). Namely, the particle cannot be 


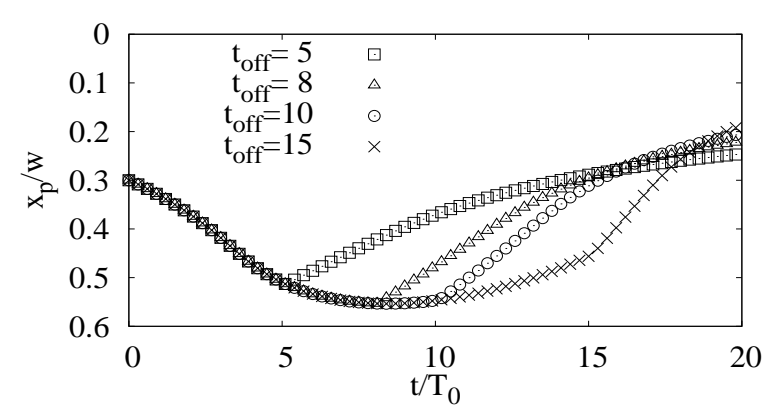

(a) Dependence of $x_{p}$ on $t\left(V_{0}=1.5 \mathrm{~V}, r_{p} / w=0.1\right)$

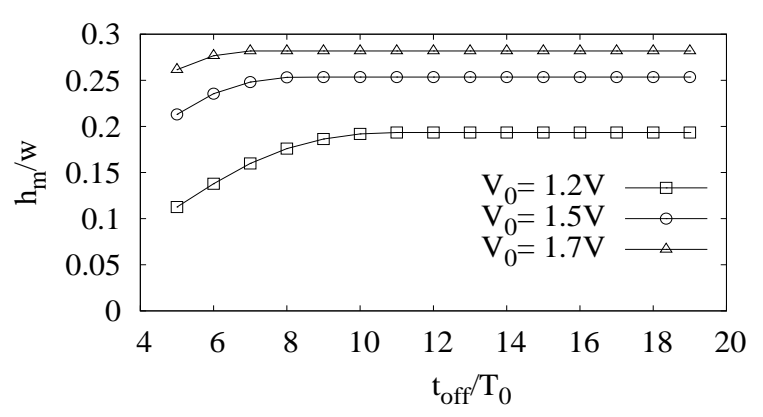

(c) Dependence of $h_{m}$ on $t_{\text {off }}\left(r_{p} / w=0.1\right)$

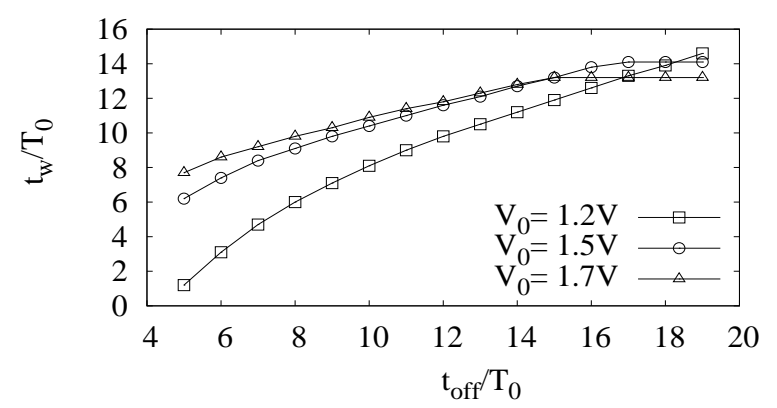

(e) Dependence of $t_{w}$ on $t_{o f f}\left(r_{p} / w=0.1\right)$

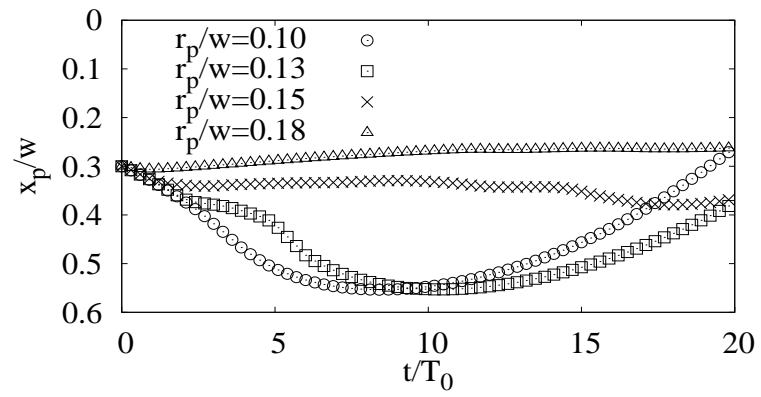

(b) Dependence of $x_{p}$ on $t\left(V_{0}=1.5 \mathrm{~V}, t_{o f f}=\infty\right)$

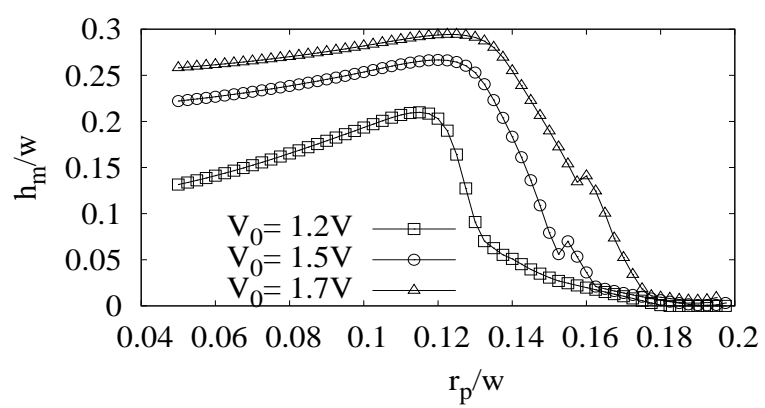

(d) Dependence of $h_{m}$ on $r_{p}\left(t_{o f f}=\infty\right)$

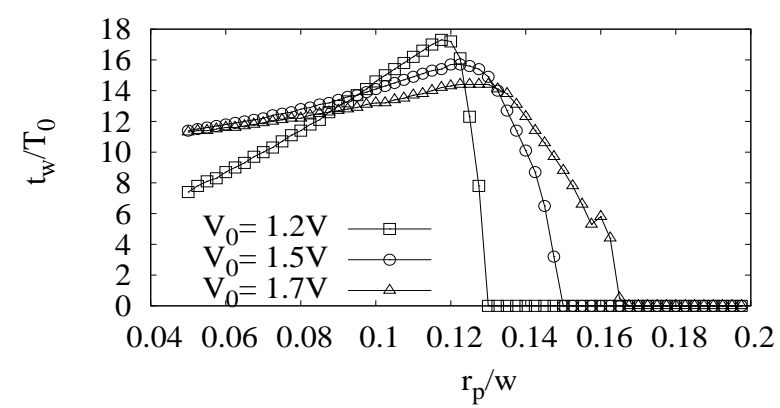

(f) Dependence of $t_{w}$ on $r_{p}\left(t_{o f f}=\infty\right)$

FIG. 3. Controllability of the keeping time and acceptable size of the particle catcher using ICEO. Here, $h_{m} \equiv x_{p}^{\max }-x_{p}^{t=0}$ is the maximum catching distance and $t_{w}$ is the keeping time; $x_{p}^{t=0} / w=0.3, d_{\min } / w=0.05, r_{s}=L-y_{e}-L_{e} \cos \theta=0.14, L / w=2.25, y_{e} / w=0.7, \nu=0.5$, $G=0.5 \mathrm{MPa}, L_{e} / w=1.5, d / w=0.07, \theta=20^{\circ}, w=100 \mu \mathrm{m}, T_{0}=1 \mathrm{~ms}, \mu=1 \mathrm{mPa} \mathrm{s}$, and $\Delta P=0 \mathrm{~Pa}$. Note that the lines [in (c) to (f)] are not analytical results but auxiliary conductors for clarifying the threshold phenomenon, while the symbols show the numerical results obtained by the BEM. 
trapped for a long time; instead, the particle is released automatically, after being caught as explained in Appendix B1. Furthermore, Fig. 3(c) shows the dependence of $h_{m}$ on $t_{\text {off }}$. In Fig. 3(c), $h_{m}$ reaches a limiting value at a large $t_{o f f}$. This is also because the deflection of the beam reaches its limit. Thus, for a rigid beam with a larger $G$, we expect that the limit $h_{m}$ will decrease under the same condition, e.g., $h_{m}^{\text {limit }} / w \simeq 0.15$ at $G=1 \mathrm{MPa}$ and $V_{0}=1.5 \mathrm{~V}$.

Figures 3(b), 3(d), and 3(f) show that the acceptable particle size is controlled in the range $0.13 \leq r_{c} / w \leq 0.17$ by adjusting the applied voltage from $V_{0}=1.2$ to $1.7 \mathrm{~V}$. Specifically, the particles of $r_{p} / w=0.10$ and 0.13 can enter the trapped region at $V_{0}=1.5 \mathrm{~V}$ and $t_{\text {off }}$, whereas the particles of $r_{p} / w=0.15$ and 0.18 cannot enter the trapped region under the same condition. Furthermore, Figs. 3(d) and 3(f) show that the threshold size can be changed by adjusting the voltage. Furthermore, as $V_{0}$ decreases the maximum $t_{w}\left(h_{m}\right)$ becomes larger (smaller), as shown in Fig. 3(f) [in Fig. 3(d)]. This is because the decrease in $V_{0}$ causes the decrease in the velocity of the pop-up motion. Note that, in Fig. 3(d), the curves of $V_{0}=1.5$ and $1.7 \mathrm{~V}$ show a small discontinuity at around $r_{p} / w=0.15$ and 0.16 . In our understanding, near the threshold radius, the increase in radius causes a decrease in the critical distance between the particle and beam edge surfaces, thus causing a rapid drawing near the edge. However, the increase in radius generally causes the increase in the shear stress $p_{s}=\mu V_{s} / d_{c}$; this usually causes the delay of the drawing phenomenon. Namely, we consider that the subtle balance causes the small discontinuity at $r_{p} \simeq r_{c}$. In fact, in the calculation of Fig. 3(d), the particle of $r_{p}=0.16$ starts the downward motion faster than the particle of $r_{p}=0.1575$ under the condition $V_{0}=1.7 \mathrm{~V}$.

Figure 4 shows the dependence of $r_{c}$ on $V_{0}$. Here, the threshold particle radius $r_{c}$ is defined as the particle radius that satisfies the condition that $h_{m} \geq 0.1$. Furthermore, the triangles, circles, and squares (dashed, solid, and dotted lines) show the numerical results obtained by the BEM [the analytical results obtained using Eq. (9)] at $G=1.0,0.5$, and $0.2 \mathrm{MPa}$, respectively. In Fig. 4, our model in Appendix B2 fairly explains the dependence of $r_{c}$ on $V_{0}$ for the rigid beams of $G=0.5$ and $1.0 \mathrm{MPa}$, although it does not explain quantitatively the behavior of the small rigidity beam of $G=0.2 \mathrm{MPa}$ since our analytical model does not consider the large diffraction in confined space. For example, the large diffraction due to the small rigidity results in a small gap distance between the beam edge and the upper wall, and it significantly increases the flow resistance for the drawing flow. 


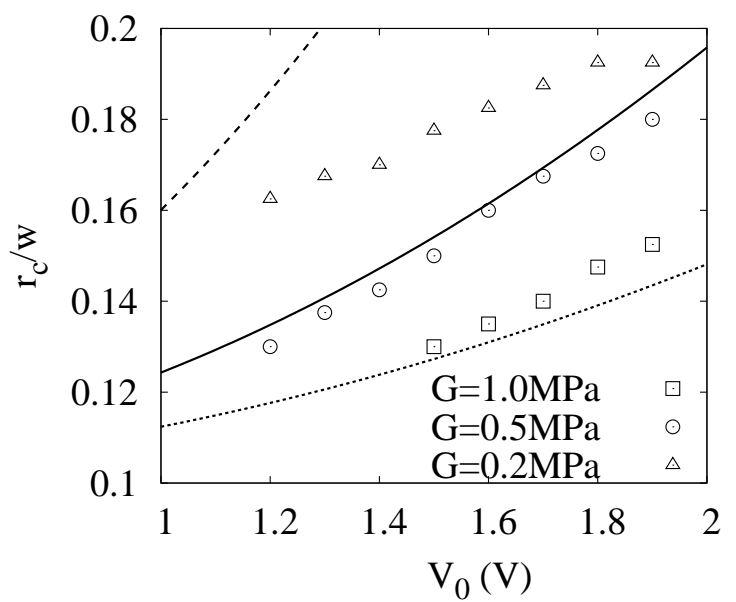

FIG. 4. Dependence of $r_{c}$ on $V_{0}$. Here, triangles, circles, and squares (dashed, solid, and dotted lines) show the numerical results obtained by the BEM [the analytical results obtained using Eq. B4] at $G=1.0,0.5$, and $0.2 \mathrm{MPa}$, respectively; $x_{p}^{t=0} / w=0.3, d_{\min } / w=0.05, r_{s}=L-y_{e}-L_{e} \cos \theta=$ $0.14, L / w=2.25, y_{e} / w=0.7, \nu=0.5, L_{e} / w=1.5, d / w=0.07, \theta=20^{\circ}, w=100 \mu \mathrm{m}, T_{0}=1 \mathrm{~ms}$, $\mu=1 \mathrm{mPa}$ s, and $\Delta P=0 \mathrm{~Pa}$.

Thus, $h_{m}$ decreases; furthermore, as explained in Sec.II-D, the accepted volume results from the volume expansion of the chamber region due to the upward motion of the beam, and thus the large volume particle of large radius cannot enter the chamber region from the beginning. Namely, we think that, by considering the hydrodynamic behaviors near the upper electrode, the model will improve significantly in the future, whereas our model probably will not improve significantly even if we consider the nonlinear bending effect, since the problem is the second issue. Thus, analytically, a lot of challenging work still remains to improve the catching theory; however, our simple model is useful for predicting the acceptable radius of the particle catcher, as the first step.

\section{Catching and releasing motions in the stream}

Figure 5 shows the catching and releasing motions in the stream with the existence of the pressure difference $\Delta P=P_{1}-P_{2}=2 \mathrm{~Pa}$. Namely, here, we demonstrate the catching and releasing motions when there is net flow of the liquid along the device channel and therefore the particle is carried away by the fluid as it would be the case in a real device. As an 
example, we set a particle of radius $r_{p}=0.1 w$ at $\left(x_{p} / w, y_{p} / w\right)=(0.3,2.0)$ at $t / T_{0}=0$ when $V_{0}=1.5 \mathrm{~V}$, in Fig. 5(a), i.e., the particle moves from the left inlet of the channel. When the particle is passing by the position of $\left(x_{p} / w, y_{p} / w\right)=(0.3,2.0)$, we apply a voltage; thus, the opening motion of the beams starts in the pressure flow, in Fig. 5(a). Then, the target particle is drawn by the drawing flow into the chamber region from the main stream region, reaching a point near the beam edge at $t / T_{0}=5[$ Fig. $5(\mathrm{~b})]$. Note that the vortex flow near the beam edge plays an essential role in drawing the particle located relatively far from the center at $x=2.25$. Then, as the beams move to the upper position, the particle is drawn into the deeper chamber region; thus, the particle is caught at $t / T_{0}=10[$ Fig. $5(\mathrm{c})]$. Then, the particle is popped up into the main stream region at $t / T_{0}=15$ [Fig. $\left.5(\mathrm{~d})\right]$. Note that the pop-up mechanism of Fig. 5(d) is mainly due to the automatic pop-up mechanism by the stoppage of motion, as mentioned in Sec. II-D, rather than to the pop-up mechanism due to the returning motion of the elastic beam, since we just switch off the applied voltage at $t / T_{0}=15$ (i.e., $t_{o f f} / T_{0}=15$ ). After that, the target particle continues to flow in the main stream again [Figs. 5(e) and 5(f)]. Figure 5 clearly shows that the catching and releasing effect is not a specific effect that occurs for the particle at the center of $x_{p} / W=2.25$ but a general effect that occurs for the particle flowing in the main stream, although there is a finite effective region for catching.

Figure 6 shows the catching region in the main stream. In Fig. 6(a), particles are caught and released when $2.0 \leq y_{p} / w$ at $x_{p} / w=0.3$. Furthermore, in Fig. 6(b), particles are caught and released when $2.5 \leq x_{p} / w \leq 3.5$ at $y_{p} / w=2.1$. Thus, we find that particles are caught and released in the range $\left|\left(y_{c}-y_{p}\right) / w\right| \leq 0.25$ and $\left(x_{c}-x_{p}\right) / w \leq 0.25$, where $\left(x_{c} / w, y_{c} / w\right)=\left(1-\frac{L_{e}}{w} \sin \theta, 2.25\right) \simeq(0.5,2.25)$ is the center position of the inlet of the catcher; i.e., the effective catching radius is approximately $r_{\text {catch }} \leq 0.25$. Here, compared with the particle radius $r_{p} / w=0.1$ and the inlet radius $r_{s}=0.14$, the effective catching radius is significantly large. Thus, we can catch particles easily without finding a suitable pin-point position, i.e., our catching device works well in many different situations of the system. 


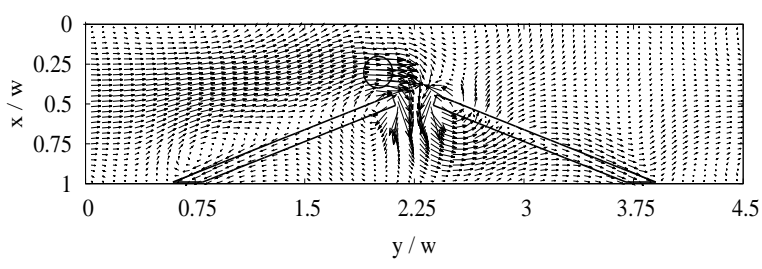

(a) Flow field at $t / T_{0}=0$

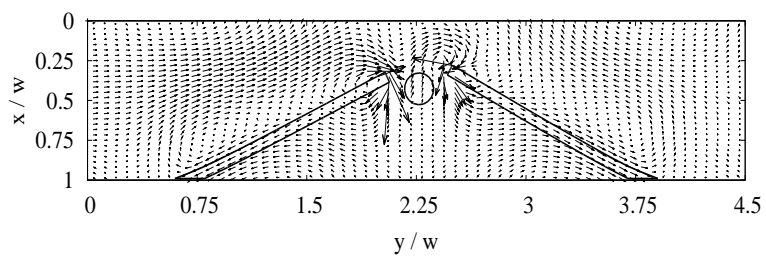

(c) Flow filed at $t / T_{0}=10$

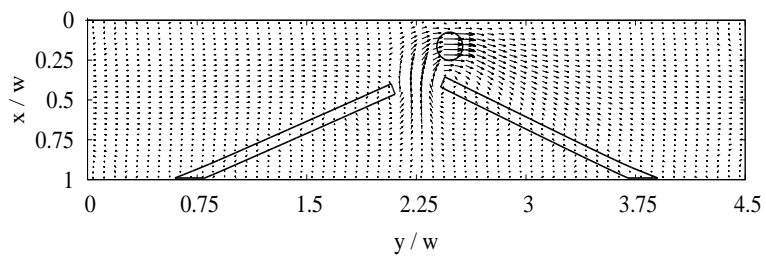

(e) Flow field at $t / T_{0}=20$

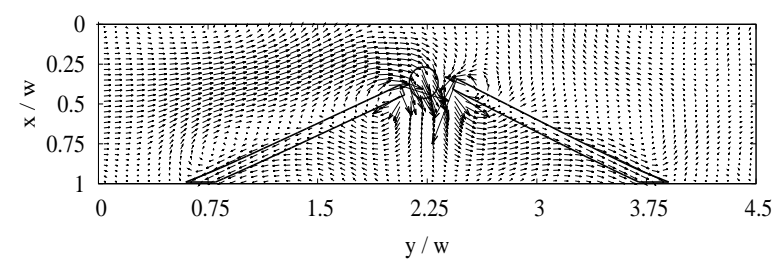

(b) Flow filed at $t / T_{0}=5$

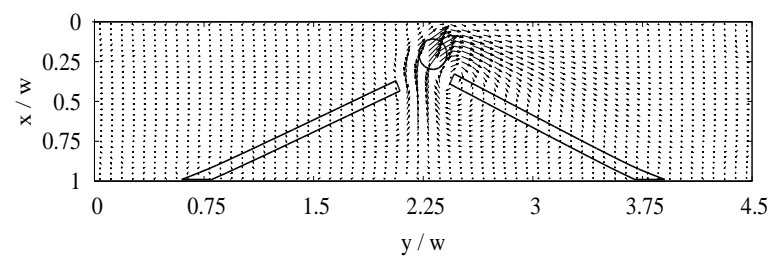

(d) Flow filed at $t / T_{0}=15$

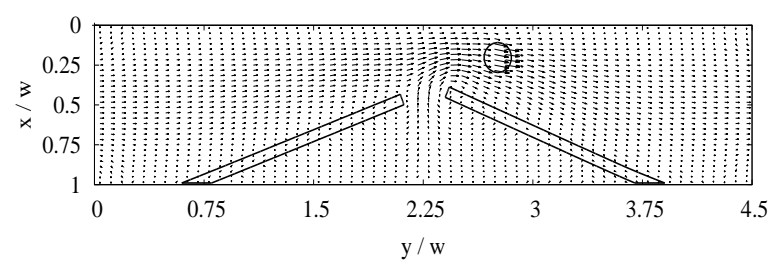

(f) Flow filed at $t / T_{0}=30$

FIG. 5. Catching and releasing motions in the stream. Here, the initial position of the particle is $\left(x_{p} / w, y_{p} / w\right)=(0.3,2.0), G=0.5 \mathrm{MPa}, V_{0}=1.5 \mathrm{~V} . r_{p} / w=0.1, t_{o f f} / T_{0}=15, d_{\min } / w=0.05$, $r_{s}=L-y_{e}-L_{e} \cos \theta=0.14, L / w=2.25, y_{e} / w=0.7, \nu=0.5, L_{e} / w=1.5, d / w=0.07, \theta=20^{\circ}$, $w=100 \mu \mathrm{m}, T_{0}=1 \mathrm{~ms}, \mu=1 \mathrm{mPa}$, and $\Delta P=P_{1}-P_{2}=2 \mathrm{~Pa}$.

\section{DISCUSSION}

\section{A. Importance of our findings}

We first propose a microfluidic particle catcher using ICEO and examined it theoretically in this paper. In particular, we find that, using ICEO a pair of conductive elastic beams having a face-to-face structure can withdraw a target particle from the main stream of a channel into the device region, keep a controlled period, and then release it into the main stream again (Fig. 1). Furthermore, we find that we can change the acceptable particle size of the catching device dynamically by changing the applied voltages. As pointed out by several researchers $[13,14,29,30]$, the catching and releasing motions are useful for future microfluidic biomedical applications. For example, although usually the microfluidic 


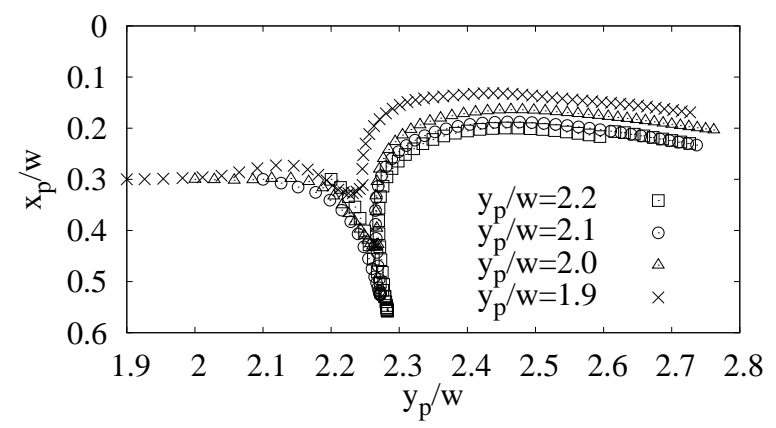

(a) Trajectory difference due to $y_{p}^{t=0} / w$

$$
\left(x_{p}^{t=0} / w=0.3\right)
$$

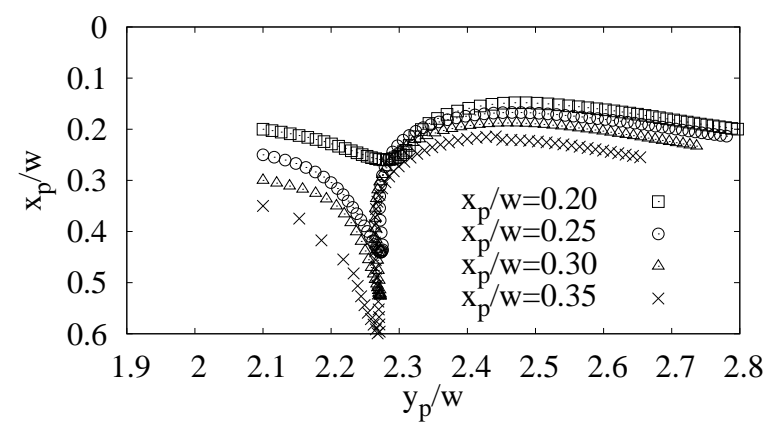

(b) Trajectory difference due to $x_{p}^{t=0} / w$

$$
\left(y_{p}^{t=0} / w=2.1\right)
$$

FIG. 6. Analysis of catching region. From the analysis, we can estimate that the catching region of our device is approximately at $0.25 \leq x_{p} / w \leq 0.35$ and $2.0 \leq y_{p} / w \leq 2.25$ for the stream of $\Delta P=2 \mathrm{~Pa}$. Here, $G=0.5 \mathrm{MPa}, V_{0}=1.5 \mathrm{~V}, r_{p} / w=0.1, t_{o f f} / T_{0}=15, d_{\min } / w=0.05$, $r_{s}=L-y_{e}-L_{e} \cos \theta=0.14, L / w=2.25, y_{e} / w=0.7, \nu=0.5, L_{e} / w=1.5, d / w=0.07, \theta=20^{\circ}$, $w=100 \mu \mathrm{m}, T_{0}=1 \mathrm{~ms}, \mu=1 \mathrm{mPa}$, and $\Delta P=P_{1}-P_{2}=2$ Pa. Furthermore, the symbols show the numerical results obtained by the BEM.

separation technique requires many channels with different diameters as well as many pumps [31], the proposed particle catcher may provide a simple system that diagnoses a target cell selectively at a high processing speed. Note that different from magnetic cilia that need a large coil, our proposed device can be miniaturized since it functions at low applied voltages $(\sim 1 \mathrm{~V})$ with simple structures, i.e., it enables the miniaturization of the entire unit including the surrounding units. Furthermore, although the catching and releasing motions in artificial 
cilia were demonstrated in previous studies [13, 14, 29, 30], external force was assumed as a given parameter. Thus, our analysis is important since it provides for the first time basic predictions in the device level. Furthermore, the size selectivity in Ref. [14] is static (i.e., the acceptable size is determined by the given parameter), whereas our proposed device has dynamic selectivity. Thus, our proposed device is definitely important for future systems in controlling the whole system at will.

\section{B. Merits of a movable polarizable electrode}

By using the direct connection between the lower electrode and the conductive elastic beam, the typical zeta potential of the edge position of the beam becomes comparable to the applied voltage and thus we can expect a large deflection of the beams, as mentioned in Ref. [23]. Since the polarizable part is the electrode, the configuration basically belongs to the ACEO configuration. Thus, this configuration has a merit of the ACEO configuration. However, different from ordinary ACEO, it is characterized by the movable polarizable electrode, i.e., it includes both ACEO and ICEO aspects and thus is more complex. Namely, the ICEO phenomenon for a movable polarizable electrode consists of four phenomena: (i) the electrophoretic phenomenon at the natural beam position, (ii) the mixed phenomenon consisting of electrophoresis and electroosmosis during an upward beam motion, (iii) the electroosmotic phenomenon at the maximum beam edge position, and (iv) the elastic phenomenon during a downward beam motion.

\section{Justification of $2 \mathrm{D}$ calculations}

Although we are inspired by the motion of cilia, our catching device is different from cilia in that it has a pair of conductive elastic plates with a face-to-face structure. Namely, the novelty of this study comes not from the cilialike structure but from the new device structure, i.e., the flapping of the face-to-face plates provides a series of catching and releasing functions above the rectangular inlet of the catching device. Thus, our main problem is intrinsically two-dimensional (2D) and our 2D calculations are justified as a first approach, although the problems that analyze the arbitrary-shaped 3D flowing objects might be interesting in the future. Furthermore, since the continuous process for spherical glass beads [11] is important 
in the novel microfluidic systems, as introduced in Sec. I, we assume the 2D particle along with the 2D face-to-face elastic beam structure. Here, although some researchers may claim that the $2 \mathrm{D}$ particle is not a spherical particle but a cylinder, the assumption of the $2 \mathrm{D}$ particle is justified since we use it only for evaluating the size selectivity of the device, i.e., since the main mechanism of size selectivity for particles results from the length $\left(2 r_{c}\right)$ of the inlet of the open state of the catcher [see Sec. II.G], the 2D simulation of the catcher using the $2 \mathrm{D}$ particle of radius $r_{p}$ clarifies the acceptable size for the 3D spherical particle at least. Furthermore, in a microfluidic setup, anisotropic particles such as cylindrical particles often change their orientation, from being parallel to the flow to being perpendicular to the flow [32]; thus, our 2D simulations indicate that the cylindrical particles of the length $\left(>2 r_{c}\right)$ are unlikely accepted by our catching device, whereas the cylindrical particles of the length $\left(<2 r_{c}\right)$ are also caught by our device. Furthermore, to focus on the main mechanism of size selectivity due to geometrical condition, we model the particle as an elastic material having the same $\nu$ and $G$ as the beams, and the electric permittivity is assumed to be the same as that of the water, as the first step. Namely, by imposing that the particle has the same permittivity as the liquid, we neglect the effect of the particle on the electric field in our setup, i.e., we neglect any possible effect of the polarization charge induced by the electrodes on the particle in the functioning of our catching device. Note that this assumption is consistent with our first aim; thus, our 2D simulations are justified as a first attempt.

\section{CONCLUSIONS}

We propose a microfluidic catcher using induced charge electro-osmosis and numerically examine its performance. By multiphysics simulations using an implicit strongly coupled calculation method between a fluid and an elastic structure along with the thin double layer approximation, we find the following: (1) Particles are drawn into the device region by the vortex flow due to the upward motion of elastic beam edges and popped up by the reverse vortex flow due to the downward motion of the edges in the transient state and by the

ICEO flow itself in the steady state. (2) The predicted drawing distance $\left(0.2 \leq h_{m} / w \leq\right.$ $0.3)$ and detention time $\left(5 \leq t_{w} / T_{0} \leq 15\right)$ are sufficiently large to use the catching and releasing motions in a microfluidic system. (3) The acceptable threshold radius is controlled 


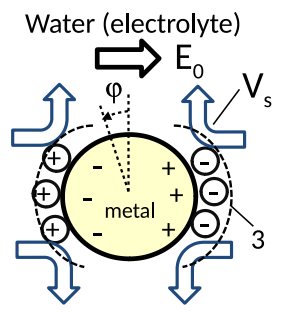

(a) ICEO vortex flow

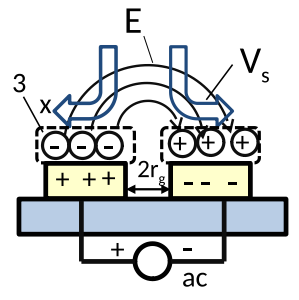

(b) ACEO vortex flow

FIG. A.0. (Color online) Bases of ICEO [15] and ACEO [16]. 3: electric double layer.

dynamically $\left(0.12 \leq r_{c} / w \leq 0.17\right)$ by changing the applied voltage $\left(V_{0} \simeq 1.2\right.$ to $\left.1.7 \mathrm{~V}\right)$. (4) The dependence of the acceptable threshold radius on applied voltage is explained fairly well by the simple theory that considers a linear elastic beam theory with the ICEO force. We believe that our device is useful for operating various substances continuously in a microfluidic channel.

\section{ACKNOWLEDGMENTS}

This work was partially supported by JSPS KAKENHI Grant Number JP16K05650.

\section{Appendix A: Bases of ICEO and ACEO}

\section{ICEO for an isolated polarizable object ("ICEO" configuration)}

Figure A.0(a) shows a typical "ICEO" configuration [15] characterized by the isolated polarizable object. In Fig. A.0(a), a circular metal cylinder of radius $a$ in electrolyte (typically water) is polarized by an external electric field $E_{0}$ and forms two electric double layers consisting of the positive and negative induced charges and counterion clouds on both sides 
of the metal cylinder. Thus, quadrupolar vortex flows are generated by the interaction of the electric double layer and the tangential electric field $E_{s}$. Here, the thickness of the electric double layer (1 to $1000 \mathrm{~nm}$ ) is often much smaller than the characteristic length. Thus, the generated electroosmotic flow velocity of the outer edge of the electric double layer is called a slip velocity $\left(V_{s}\right)$, even though the zero velocity boundary condition is assumed on a real metal surface (i.e., the inside edge of the double layer). By using the HelmholtzSmoluchowski formula assuming a thin double-layer approximation, we can describe $V_{s}$ as

$$
\boldsymbol{V}_{s}=-\frac{\epsilon \zeta}{\mu} \boldsymbol{E}_{\mathrm{s}}
$$

where $\epsilon\left(\sim 80 \epsilon_{0}\right)$ is the dielectric permittivity of the solvent (typically water), $\epsilon_{0}$ is the vacuum permittivity, $\mu(\sim 1 \mathrm{mPa} \mathrm{s})$ is the viscosity, and $\zeta\left(=\phi_{i}-\phi_{o}\right)$ is the zeta potential. Note that $\phi_{i}(=0)$ and $\phi_{o}$ are the potentials of the inside and outside edges, respectively, of the electric double layer. Thus, by using the no-flux electrostatic boundary condition, we can calculate $\phi_{o}$ and $E_{s}$ around the circular cylinder as $\phi_{o}=2 E_{0} a \cos \varphi$ and $E_{s}=-2 E_{0} \sin \varphi$ [33]. Therefore, the $V_{s}$ of the circular cylinder is described as $V_{s}=2 U_{0} \sin 2 \varphi$, where

$$
U_{0}=\frac{\epsilon a E_{0}^{2}}{\mu}
$$

is a represented flow velocity of ICEO. Interestingly, as predicted by Bazant and Squires [15] and experimentally examined by Gangwal et al. [25], the Janus particle that has both metal and insulator surfaces can move in electrolyte at the velocity

$$
U_{\text {janus }}=\frac{9}{64} U_{0}
$$

because of the unbalanced ICEO flows, i.e., the generated force due to the ICEO flow is

$$
F_{\text {janus }}=6 \pi a \mu \frac{9}{64} U_{0} \sim \pi a \mu U_{0}
$$

on the basis of Stokes' law. Note that, under the low Reynolds number condition, the given total force of the object always balances with the flow resistance; this situation is very different from that in the ordinary world. Furthermore, Stokes' law tells us that, when $F_{\text {janus }}$ induces $U_{\text {janus }}$ in the same direction as $F_{\text {janus }}$, the opposite force (due to the flow resistance), the strength of which is proportional to $U_{\text {janus }}$, is generated and it balances with $F_{\text {janus }}$. Here, $F_{\text {janus }}$ is the force to fix the particle as a result of the action-reaction law concerning the Coulomb force that generates an ICEO flow. 


\section{ICEO for a fixed polarizable electrode ("ACEO" configuration)}

Figure A.0(b) shows a typical "ACEO" configuration [16] characterized by a fixed polarizable electrode. In Fig. A.0(b), by applying an ac voltage of amplitude $V_{0}$, the electrode surface is polarized and has a positive or negative charge; then, the counterions are attracted to the surface and form an electric double layer. Thus, by the interaction between the electric double layer and the tangential electric field $E_{s}$, a pair of electro-osmotic flows are generated in the opposite directions on both electrodes. Here, the background physics of ACEO is the same as that of ICEO configurations. However, practically, the ACEO configuration has some merits over the ICEO configuration. Firstly, since half of the applied voltage is screened by the counterions in ACEO, we can take a much larger zeta potential (i.e., $\zeta^{\text {aceo }} \simeq V_{0} / 2$ and typically $0.5 \mathrm{~V}$ ) than that in ICEO configurations (i.e., $\zeta^{\text {iceo }} \simeq 2 a E_{0}$ and typically $0.1 \mathrm{~V}$ ), where $W$ is the distance of the parallel electrodes that generate the electric field $E_{0}=V_{0} / W$; the typical $V_{0}, a$, and $W$ are $1 \mathrm{~V}, 0.1 \mathrm{~W}$, and $100 \mu \mathrm{m}$, respectively. Secondly, since we need not put polarizable objects between electrodes in ACEO, we can also take a much larger tangential electric field $\left(E_{s}^{a c e o} \simeq V_{0} / 2 r_{g}\right)$ than that in ICEO configurations $\left(E_{s}^{i c e o} \simeq V_{0} / W\right)$ by setting a small $2 r_{g}(\ll W)$, where $2 r_{g}$ is a gap distance between planar electrodes. Thus, the slip velocity of ACEO is expected to be

$$
V_{s}^{a c e o} \simeq\left(\frac{1}{4 r_{g}}\right) \frac{\epsilon V_{0}^{2}}{\mu}
$$

and under the condition that $4 r_{g} \ll W$ and $2 a / W<1$, it is much larger than that of ICEO configurations,

$$
V_{s}^{i c e o} \simeq\left(\frac{2 a}{W^{2}}\right) \frac{\epsilon V_{0}^{2}}{\mu} .
$$

Furthermore, since the capacitance $C_{D}$ and the accumulated charge $q_{s}$ of the electric double layer on the electrode for a unit area at $V_{0}$ are described as $C_{D}=\epsilon / \lambda_{D}$ and $q_{s}=$ $C_{D}\left(V_{0} / 2\right)$, respectively, Eq. A6 is rewritten as [16]

$$
V_{s}^{\text {aceo }} \simeq \frac{\lambda_{D}\left(\frac{\epsilon}{\lambda_{D}}\right)\left(\frac{V_{0}}{2}\right) E_{s}}{\mu} \simeq \frac{\lambda_{D} q_{s} E_{s}}{\mu} \simeq \frac{\lambda_{D} f_{c}^{\text {aceo }}}{\mu},
$$

where $\lambda_{D}$ is the Debye screening length and $f_{c}^{a c e o}=q_{s} E_{s}$ is a Coulomb force parallel to the electrode surface for a unit area. Note that, because of the action-reaction law, the reaction force

$$
f_{r}^{a c e o}=-f_{c}^{a c e o}=-\mu \frac{V_{s}^{\text {aceo }}}{\lambda_{D}}
$$


acts on the electrode in the opposite direction, i.e., the force of ICEO is equivalent to the shear stress characterized by $V_{s}$ and $\lambda_{D}$ in the double layer and the opposite force acts on the electrode, although in the ordinary ACEO configurations the electrodes are always fixed in space.

\section{Appendix B: Analytical models}

\section{Model of catching and releasing effects using a face-to-face elastic beam con-} figuration

From the argument of Eq. A8, if we assume that the dominant ICEO (ACEO) force only acts on the beam edge of thickness $d$, the motion of the single elastic beam is described by the kinetic equation

$$
f_{r}^{a c e o} L_{e} d-R_{r} \dot{\theta}-T_{e} \simeq 0\left(=I_{b} \ddot{\theta}\right)
$$

where $\dot{\theta}$ is angular velocity, $\ddot{\theta}$ is angular acceleration, $R_{r}$ is the resistance coefficient for rotation, $T_{e}$ is the elastic torque of the beam, $I_{b}$ is the inertia moment of the beam, and $L_{e}$ is the beam length. Namely, (1) since $T_{e} \simeq 0$ in the initial state, the ACEO torque balances only with the rotational flow resistance $\left(R_{r} \dot{\theta}\right)$, i.e., the ACEO torque is mainly used for opening the beams and the fluid flow velocity due to ACEO is small. (2) During the upward motion, the ACEO torque balances with the rotational flow resistance and elastic torque. Thus, as the elastic torque increases, the angular velocity of the beams decreases and the fluid flow velocity due to ACEO becomes large; the ACEO torque is used both for opening the beams and for the generation of the drawing flow into the chamber region enclosed partially by the face-to-face beams. Note that the upward motion of the beams helps the accumulation of fluid (and a particle) by the drawing flow; this mechanism enables a catching effect due to the face-to-face beam configuration. (3) In the halt state, the ACEO torque balances only with the elastic torque, i.e., all the ACEO torque is used for the generation of the pop-up fluid flow at the center position since the downward ICEO flow at the beam edge position can no longer be accumulated in the chamber region because of the stoppage of the beams. Thus, an automatic releasing effect occurs. (4) During the downward motion, the elastic torque balances with the rotational flow resistance. This situation also causes the pop-up fluid flow at the center, since the fluid in the chamber region is squeezed out 
by the downward motion of the elastic beams. Consequently, the catching and releasing effects can be realized by the face-to-face configuration with the ICEO (ACEO) phenomena. Moreover, in Fig. 1(d), the series of complex phenomena provide the ideal mechanism for a novel high-throughput continuous processing method, i.e., a particle in the main stream region can be drawn into the chamber region between the beams and stay during a short period in the region; then, it is popped up into the main stream region again.

Note that, in Eq. B1, $f_{r}^{a c e o}$ is not a body force such as a Coulomb force of the double layer but a reaction force fixed on the beam, as a result of the action-reaction law, as explained in Appendix A2. Furthermore, for the calculation of the rigid particle in a fluid at small $R e$, we usually consider the force and torque balance equations; i.e., $\int_{S_{p}} \boldsymbol{f} d l+\boldsymbol{F}_{t}^{e x t}=0$ and

$\int_{S_{p}} \boldsymbol{x} \times \boldsymbol{f} d l+\boldsymbol{T}_{t}^{e x t}=0$, where $S_{p}$ denotes the surface of the particle, $\boldsymbol{F}_{t}^{e x t}$ is a total external force (such as DEP and repulsion forces), and $\boldsymbol{T}_{t}^{e x t}$ is a total external torque (such as DEP and repulsion torques); the balance equations make sure that the net force and torque are zero at $\boldsymbol{F}_{t}^{e x t}=\boldsymbol{T}_{t}^{e x t}=\mathbf{0}$. However, since our problem is the FSI problem, we need to consider Eq. (4) based on the action-reaction law on the boundary between fluid and solid. Implicitly, we also consider that $\boldsymbol{f}_{e}=\boldsymbol{f}^{\text {wall }}$ at $x / w=1$ on the basis of the action-reaction law. Thus, all of the surface tractions around the beam locally balance with the surrounding surface tractions of the fluid and wall, although it is a common situation of the stress-strain calculations of elastic (solid) objects.

\section{Model of the size-selectable separation effects}

By considering the Lorentz reciprocal theorem $[23,34,35]$, we approximate an effective ICEO force of unit thickness at $\boldsymbol{x}_{p}$ in the upper direction of the beams in the presence of an electric field as

$$
F^{I C E O} \simeq \mu V_{s}^{e d g e}
$$

where $V_{s}^{\text {edge }}\left(\simeq c_{e} U_{w}\right)$ is the edge slip velocity, $U_{w}=\epsilon w E_{0}^{2} / \mu$ is a characteristic velocity of ICEO of the channel, and $c_{e}(\simeq 3)$ is a shape factor related to the strength of the local electric fields at the beam edge. By considering the linear beam theory, we can approximate the deflection of the beams [23] as

$$
\delta^{\text {beam }} \simeq \frac{c_{A} F^{I C E O} L_{e}^{3}}{3 E I}
$$


where $E=2 G(1+\nu)$ is Young's modulus, $G$ is the rigidity modulus, $\nu$ is the Poisson ratio, $I=d^{3} / 12$ is the moment of the inertia for the rectangular cross section of unit thickness, and $c_{A}(\simeq 12.5)$ is the correction coefficient for a real nonlinear problem. From the geometrical conditions, we obtain an acceptable threshold particle size as

$$
r_{c} \simeq r_{s}+r_{e}-r_{a}
$$

where $r_{s}=L-y_{e}-L_{e} \cos \theta$ is a static hole radius, $r_{e}=\delta^{\text {beam }} \sin \theta$ is an expanded hole radius due to a ICEO flow, and $r_{a}=0.8 d_{\min }$ is an apparent repulsive radius.

\section{Model of a repulsion force}

We phenomenologically assume that the repulsive force between the elastic beams and the target particle is

$$
\boldsymbol{f}_{b p}=-f_{0} \frac{d_{\min }-d_{b p}}{d_{b p}} \frac{\boldsymbol{r}_{b p}}{\left|\boldsymbol{r}_{b p}\right|}\left(a t d_{b p}<d_{\min }\right)
$$

where $\boldsymbol{r}_{b p}=\boldsymbol{r}_{p}-\boldsymbol{r}_{b}, \boldsymbol{r}_{p}$ and $\boldsymbol{r}_{b}$ are position vectors of the particle and beam surfaces, $d_{b p}=$ $\left|\boldsymbol{r}_{b p}\right|$ is the distance between the particle and beam surfaces, $d_{\min }(=0.05 \mathrm{w})$ is an effective length of the repulsion force, and $f_{0}(=20 \mathrm{~Pa})$ is a proportionality constant. Note that Eq. B5 is implemented by considering that $\boldsymbol{f}_{r e p}=\boldsymbol{f}_{b p}$ in Eq. (4) in the numerical calculations. Thus, implicit and stable calculations are realized even if we use this phenomenological force. In other words, since Eq. B5 is implemented so that it realizes a complete local force balance among $\boldsymbol{f}_{e}^{\prime}, \boldsymbol{f}_{e}^{\prime}, \boldsymbol{f}_{r e p}$, and $\boldsymbol{f}_{f}$ at each time step at low Reynolds numbers, it prevents the unphysical invasion of the particle into the beam region. Thus, Eq. B5 is essential to calculate the threshold of particle size, and without it the calculation of near threshold will break down.

Similar repulsive forces are commonly used for the calculation at low Reynolds numbers to prevent mechanical contact [36, 37]; for example, Saintillan et al. [37] performed multifiber simulations by assuming the strong short-range repulsive forces between fibers. Although the situation is different from our problem, the repulsive force can be described as $\boldsymbol{f}_{b p}=$ $-\frac{f_{0}}{d_{\min }} \frac{e^{-d_{b p} / d_{\min }}}{1-e^{-d_{b p} / d_{\min }}} \boldsymbol{n}$ at $d_{b p}<d_{\min }$. Thus, when $d_{b p}=\left|\boldsymbol{r}_{d p}\right|$ approaches zero (i.e., the particle is moving close to the beam surface), $\left|\boldsymbol{f}_{b p}\right|$ tends to approach infinity in both the above equation and Eq. B5; thus, the particles never touch the beam surface. Although the behavior is 
unphysical, it is customary to use these repulsive forces to prevent the mechanical contact causing a serious breakdown of the numerical calculations. However, the repulsion force is large near the surface $\left(0<d_{b p} \ll d_{\text {min }}\right)$ but small at $d_{b p} \simeq d_{\text {min }}$; thus, it requires an extremely small time step. In addition, the artificial repulsive force has unpreferable infinite effective distance. Therefore, in Eq. B5, we limit the area of influence within $d_{b p}<d_{\min }$ and avoid the generation of the extremely large repulsive force. As a result, with a reasonable time step period, we could prevent the particle from approaching the beam. Specifically, the condition $d_{\text {min }}=0.05 w$ and $f_{0}=20 \mathrm{~Pa}$ is selected so that it prevents the contact between the particle and the fluid within a suitable calculation time. Of course, a small $d_{\min } / w$ is preferable but it has a trade off, i.e., a long calculation time. Thus, our calculations are justified as a first approach. Furthermore, when the particle of $r_{p} \simeq r_{c}$ passes the inlet of the catcher, the distance between the beam edge and the surface of the particle is very small. Thus, if we select the critical distance $d_{c}$ as $d_{\text {min }} / 2$, the shear stress is estimated as $p_{s} \simeq \mu V_{s} / d_{c}=13.3$ $\mathrm{Pa}$ at $V_{0}=1.19 \mathrm{~V}$ and $w=100 \mu \mathrm{m}$, and the $p_{s}$ is expected to be compatible with the approaching pressure, since $\left|\boldsymbol{f}_{b p}\right|=f_{0}=20 \mathrm{~Pa}$ at $d_{b p}=d_{\min } / 2=0.025 \mathrm{w}$ in Eq. B5. Thus, to prevent serious contact, the conditions $d_{c}=0.025 \mathrm{w}$ and $f_{0}=20 \mathrm{~Pa}$ are reasonable as a first attempt, even though $d_{c}=0.025 \mathrm{w}$ may tend to underestimate the threshold value $r_{c}$.

[1] G. M. Whitesides, Nature 442, 368 (2006).

[2] T. Kulrattanarak, R. van der Sman, C. Schron, and R. Boom, Adv. Colloid Interface Sci. 142, 53 (2008).

[3] D. Reguera, A. Luque, P. S. Burada, G. Schmid, J. M. Rubí, and P. Hänggi, Phys. Rev. Lett. 108, 020604 (2012).

[4] M. Balvin, E. Sohn, T. Iracki, G. Drazer, and J. Frechette, Phys. Rev. Lett. 103, 078301 (2009).

[5] M. Yamada and M. Seki, Lab Chip 5, 1233 (2005).

[6] J. D. Adams, P. Thvoz, H. Bruus, and H. T. Soh, Applied Physics Letters 95, 254103 (2009).

[7] M. MacDonald, G. Spalding, and K. Dholakia, Nature 426, 421 (2003).

[8] T. A. J. Duke and R. H. Austin, Phys. Rev. Lett. 80, 1552 (1998).

[9] J. A. Davis, D. W. Inglis, K. J. Morton, D. A. Lawrence, L. R. Huang, S. Y. Chou, J. C. 
Sturm, and R. H. Austin, Proc. Natl. Acad. Sci. U.S.A. 803, 14779 (2006).

[10] K. J. Morton, K. Loutherback, D. W. Inglis, O. K. Tsui, J. C. Sturm, S. Y. Chou, and R. H. Austin, Lab Chip 8, 1448 (2008).

[11] S. H. Chung, C. Baek, V. T. Cong, and J. Min, Biosensors and Bioelectronics 67, 625 (2015), special Issue: $\{$ BIOSENSORS\} 2014.

[12] J. den Toonder and P. Onck, Trends in Biotechnology 31, 85 (2013).

[13] A. Bhattacharya, G. A. Buxton, O. B. Usta, and A. C. Balazs, Langmuir 28, 3217 (2012).

[14] A. Tripathi, A. Bhattacharya, and A. C. Balazs, Langmuir 29, 4616 (2013).

[15] M. Z. Bazant and T. M. Squires, Phys. Rev. Lett. 92, 066101 (2004).

[16] A. Ramos, H. Morgan, N. G. Green, and A. Castellanos, Journal of Colloid and Interface Science 217, 420 (1999).

[17] A. Ramos, A. Gonzalez, A. Castellanos, N. G. Green, and H. Morgan, Phys. Rev. E 67, $056302(2003)$.

[18] M. Z. Bazant, M. S. Kilic, B. D. Storey, and A. Ajdari, Advances in Colloid and Interface Science 152, 48 (2009).

[19] F. Ziebert, M. Z. Bazant, and D. Lacoste, Phys. Rev. E 81, 031912 (2010).

[20] J. P. Urbanski, T. Thorsen, J. A. Levian, and M. Z. Bazant, Appl. Phys. Lett. 89, 143508 (2006).

[21] H. Sugioka, Phys. Rev. E 78, 057301 (2008).

[22] H. Sugioka, Phys. Rev. E 81, 036301 (2010).

[23] H. Sugioka, Phys. Rev. Applied 3, 064001 (2015).

[24] H. Sugioka, Phys. Rev. E 81, 036306 (2010).

[25] S. Gangwal, O. J. Cayre, M. Z. Bazant, and O. D. Velev, Phys. Rev. Lett. 100, 058302 (2008).

[26] A. Ramos, H. Morgan, N. G. Green, and A. Castellanos, Journal of Physics D: Applied Physics 31, 2338 (1998).

[27] H. Sugioka, Journal of the Physical Society of Japan 85, 104001 (2016).

[28] T. M. Squires and M. Z. Bazant, Journal of Fluid Mechanics 560, 65 (2006).

[29] H. Masound and A. Alexeev, Soft Matter 7, 8703 (2011).

[30] R. Ghosh, G. A. Buxton, O. B. Usta, A. C. Balazs, and A. Alexeev, Langmuir 26, 2963 (2010).

[31] M. Yamada, K. Kano, Y. Tsuda, J. Kobayashi, M. Yamamoto, M. Seki, and T. Okano, 
Biomed Microdevices 9, 637 (2007).

[32] M. Trebbina, D. Steinhauser, J. Perlich, A. Buffet, S. V. Roth, W. Zimmermanne, J. Thielef, and S. Frster, PNAS 110, 6706 (2013).

[33] T. M. Squires and M. Z. Bazant, J. Fluid Mech. 509, 217 (2004).

[34] H. Sugioka, Colloids and Surfaces A: Physicochem. Eng. Aspects 376, 102 (2011).

[35] M. C. Fair and J. L. Anderson, J. Colloid Interface Sci. 127, 388 (1989).

[36] J. Brady, Annu. Rev. Fluid Mech. 20, 111 (1988).

[37] D. Saintillan, E. Darve, and E. S. G. Shaqfeh, Phys. Fluids 17, 033301 (2005). 